\title{
Sindeleros
}

\section{Diseño universal en la educación infantil. Reflexiones frente a su implementación e implicación para el proceso de enseñanza-aprendizaje ${ }^{1}$}

\author{
Universal design in children's education. Reflections on its \\ implementation and implication for the teaching-learning process
}

\author{
Autora: \\ Nátaly Restrepo Restrepo2 \\ Recibido: 28 de febrero de 2018 \\ Aprobado: 23 de abril de 2018
}

\section{Resumen}

El diseño universal del aprendizaje, como modelo que se fundamenta en eliminar las barreras para el acceso al aprendizaje y la participación, es un eje de análisis para comprender las relaciones que se establecen entre docente, estudiante y aprendizaje en los escenarios educativos de la educación infantil; la vinculación y relación entre niño-niña-docente, agente educativo están interpeladas por múltiples prácticas y estrategias que en el hacer educativo se transforman en procesos de integración, inclusión, exclusión, o segregación, por ello se lleva a cabo un proyecto pedagógico investigativo con enfoque cualitativo acción participativa. Los resultados permiten identificar cómo la implementación de enfoques como el diseño universal del aprendizaje favorece la participación efectiva y el logro de aprendizajes de los niños y niñas, se resaltan las implicaciones que se deben tener en cuanto a la comprensión de la diversidad, la práctica pedagógica-educativa, los aprendizajes y la participación de los niños en la educación infantil. 
Palabras claves: Aprendizaje, process will be transform into práctica pedagógica, educación integration, inclusion, exclusion or infantil, infancias, enseñanza

\begin{abstract}
The universal design of learning as a model that is based on eliminating barriers to access, learning and participation, is an axis of analysis to understand the relationships that are established between teacher, student, learning in the educational scenarios of early childhood education; the relationship and relationship between boy-girl-teacher, educational agent is interpellated by multiple practices and strategies that in the educational
\end{abstract} segregation processes. The results the project research of a qualitative nature make it possible to identify how the implementation of approaches such as the universal design of learning favors the effective participation and children's learning achievement, highlighting the implications that must be had regarding the understanding of diversity, pedagogical-educational.

Keywords: Learning, pedagogical practice, childhood education, infancies, teaching

\title{
Introducción
}

El diseño universal del aprendizaje (DUA) en la educación es un tema que en los últimos años ha sido eje de gran interés para el campo educativo: las construcciones socioeducativas en relación con la diversidad, la diferencia, la inclusión, la exclusión, la flexibilización de los currículos y el mejoramiento de los procesos de enseñanzaaprendizaje asientan una variedad de perspectivas que constituyen un reto significativo para los sistemas educativos; hay que decir que en la actualidad las desigualdades se asocian a fenómenos relacionados con la calidad de la educación, especialmente para aquellos grupos excluidos que han enfrentado múltiples barreras en la participación educativa, donde la pertinencia curricular, la calidad de la enseñanza y la calidad de los aprendizajes han sido ejes transversales para la discusión por las brechas existentes entre lo político, lo social y lo educativo.

El diseño universal del aprendizaje se aborda en el marco de la educación inclusiva como medio para eliminar las barreras y fomentar aprendices expertos en un entorno de enseñanza. El DUA significa un nuevo marco para el currículo cuyo fin es dar respuesta a la diversidad maximizando las oportunidades de aprendizaje y la participación de los sujetos; en los últimos años, son varias las investigaciones que se han llevado a cabo para analizar su implementación en el ámbito educativo, por ejemplo, autores como Oquendo y Benavides (2017), en su estudio "Estrategias didácticas para la incorporación del diseño universal para el aprendizaje en la escuela rural", encuentran que algunos factores asociados a la desmotivación de los estudiantes se dan en relación con los contenidos propuestos y las metodologías empleadas, por ello buscan mejorar los procesos de enseñanza-aprendizaje mediante este enfoque y han encontrado resultados positivos. 
Otros autores (Berquist y Moore, 2014; Johnson y Mundschenk, 2014; Katz, 2014; Sánchez, Díez y Martín, 2015) analizan las metodologías docentes siguiendo los principios del diseño universal en la educación formal y universitaria, y demuestran que el desarrollo de currículos basados en el DUA favorece la puesta en marcha de estrategias inclusivas y la creación de contenidos de aprendizaje más accesibles para todos los estudiantes, asimismo identifican que una de las dificultades es ofrecer solo una opción en el acceso a las actividades.

El diseño como enfoque metodológico es una demanda que además está propuesta desde las orientaciones políticas en América latina, orientaciones como: la declaración de Jomtien (Unesco, 1990) y el marco de acción Dakar (2000); la Cumbre Mundial a Favor de la Infancia (Unicef, 1990); la Cuarta Reunión Ministerial Americana sobre Infancia y Política Social (1998); los Objetivos de Desarrollo del Milenio (2007); las metas OEI (OEI, 2021); los informes de la OCDE (2016); el Foro Mundial de Incheon sobre la Educación (2015), entre otros, refieren pronunciamientos que orientan y dan línea frente al desarrollo de procesos educativos en cuanto al acceso, la permanencia y los procesos de enseñanza-aprendizaje en la educación inicial, formal y universitaria. A nivel nacional la atención a la diversidad, específicamente en el campo de la educación, ha estado sujeta a las construcciones legislativas y normativas establecidas por la Ley 1618 de 2013, en concordancia con las normas que forman parte del bloque de constitucionalidad (Decreto1421 de 2017). La prioridad se ha mediado por garantizar atenciones de calidad enmarcadas en los principios de la educación inclusiva de calidad, la diversidad, la pertinencia, la participación, la equidad y la interculturalidad, su objetivo se centra en favorecer el acceso, la participación y el reconocimiento de los derechos de los grupos poblacionales en los diferentes entornos.

En el marco de la educación inicial y la atención integral a la primera infancia en la política de cero a siempre, por ejemplo, la atención integral se asume desde una perspectiva diferencial que actúa intencionadamente sobre los entornos para transformar situaciones de discriminación en razón de las diferencias, se toma en consideración la diversidad de los niños y niñas de acuerdo con su cultura, pertenencia étnica y contexto (Ley 1804 de 2016). Es necesario mencionar que el reconocimiento de la atención a la diversidad se ha venido configurando desde una perspectiva de derechos y un enfoque de desarrollo humano, dado que se reconoce a los niños y niñas como sujetos de derechos en permanente evolución, con una identidad específica que debe ser respetada y valorada como parte esencial del desarrollo; los niños y las niñas son seres únicos con formas propias de aprender y de expresar lo que piensan; son "seres activos que se desenvuelven en la vida social y que no solo construyen su propia subjetividad a partir de las interacciones que establecen, sino que cuentan con capacidades para expresar y darle sentido a su propia vida" (Consejería Presidencial para la Primera Infancia, 2013, p. 100)

No obstante, es preciso decir que, aunque se asume una perspectiva de derechos, algunas investigaciones evidencian que es limitada la participación de los niños y niñas menores de 6 años de zonas rurales o en situación de vulnerabilidad por etnia, 
situación económica, discapacidad, alerta en el desarrollo o situación cultural. Bernal (2014), por ejemplo, establece que en Colombia, a pesar del avance importante en la ampliación del acceso, solo la mitad de los niños de 3 años y tres cuartas partes de los niños de 4 años de edad se benefician de los servicios de educación inicial en el marco de la atención integral a la primera infancia (EIAIPI). Los niños de las familias menos favorecidas y en las zonas rurales tienen índices de participación mucho más bajos y enfrentan mayores retos de acceso a los servicios de la EIAIPI (Bernal, 2014; MEN, 2015).

En correspondencia con estas cifras, el Ministerio de Educación desde sus orientaciones técnicas administrativas y pedagógicas establece acciones y estrategias que buscan eliminar las barreras e incrementar las oportunidades de aprendizaje; una de ellas se sitúa en la implementación de propuestas pedagógicas basadas en los principios propuestos en la Guía para el Diseño Universal del Aprendizaje (DUA), con la cual se busca el desarrollo de propuestas educativas que favorezcan los desarrollos de los niños y niñas y garanticen los ajustes razonables requeridos en su proceso educativo.

Bajo esta premisa, y reconociendo la importancia que tienen las nuevas construcciones que se han tejido frente a la diversidad y la identidad en el marco pedagógico y didáctico, se busca analizar cómo la implementación de enfoques como el diseño universal del aprendizaje favorece la participación efectiva y el logro de aprendizajes de los niños y niñas en la educación infantil. Este análisis pretende generar algunas reflexiones frente a su implementación e implicación para el proceso de enseñanzaaprendizaje en la educación infantil.

\section{Metodología}

Con el fin de alcanzar los objetivos propuestos, este proyecto pedagógico investigativo se enmarcó en la modalidad de investigación interactiva. La investigación-acción participativa ayuda a comprender los procesos de enseñanza como procesos de investigación, es una forma de comprender la práctica didáctica a partir de la cual intentamos mejorarla. No solamente ayuda para resolver los problemas de la práctica, sino que es un proceso para problematizarla, y poder así reorientarla (Contreras, 1994). El proyecto contó con una propuesta didáctica que se trabajó a partir de los principios del enfoque del diseño universal del aprendizaje:

1. Principio I. Proporcionar múltiples formas de representación de la información y los contenidos (el qué del aprendizaje), ya que los alumnos son distintos en la forma en que perciben y comprenden la información.

2. Principio II. Proporcionar múltiples formas de expresión del aprendizaje (el cómo del aprendizaje), puesto que cada persona tiene sus propias habilidades estratégicas y organizativas para expresar lo que sabe.

3. Principio III. Proporcionar múltiples formas de implicación (el porqué del aprendizaje), de forma que todos los alumnos puedan sentirse comprometidos y motivados en el proceso de aprendizaje. 
El proyecto se implementó por medio de diferentes fases: la primera de caracterización, a partir de la implementación de una guía de observación se buscó caracterizar cada uno de los principios del diseño universal del aprendizaje y las percepciones de los docentes y agentes educativos, lo que posibilitó la descripción del problema y dio paso a la fase dos, etapa de diseño y planeación de la propuesta pedagógica, teniendo presente los principios establecidos por el diseño universal (representación, expresión, implicación), aplicados en la fase tres, en la que se desarrolló la propuesta y a la cual se ajustaron de acuerdo con cambios y necesidades específicas de las instituciones educativas. En esta fase se realiza la recolección de la información con instrumentos como el diario pedagógico, la matriz de análisis de diseño universal y la guía de observación; en la fase 4 se hace la sistematización y triangulación de los instrumentos y se estructuran los resultados. Finalmente, en la fase 5 se analizan y discuten los resultados y las conclusiones.

Esta implementación se realizó en instituciones de educación inicial y educación formal, específicamente en seis instituciones caracterizadas de la siguiente forma: dos instituciones de educación formal (primaria), dos instituciones de educación inicial (grado transición), y dos fundaciones sin ánimo de lucro que atienden a población en situación de vulnerabilidad con metodologías multigrado. La población participante fueron niños y niñas entre los 4 y 15 años de edad, considerando que los niveles cognitivos de algunos de ellos por su condición de discapacidad no corresponden a su ciclo de edad, la población con la que se trabaja son niños y niñas con extra edad, dificultades comportamentales, discapacidad psicosocial, sistémica y cognitiva, y niños y niñas "regulares". Para recoger la información se aplicaron técnicas e instrumentos de investigación como la guía de observación, el diario pedagógico, la matriz de análisis del diseño universal y la comunicación no verbal, además de consentimientos informados para el trabajo con los niños y el registro fotográfico. Para la sistematización se realizó la triangulación de los instrumentos del diario pedagógico, la matriz de diseño universal del aprendizaje, mediante una codificación de la información abierta axial, y posteriormente se realizó una agrupación de códigos según las características comunes y se establecieron gráficos que ayudaran a interpretar los datos e identificar las subcategorías.

\section{Resultados}

Después de la aplicación de las propuestas pedagógicas y los análisis de la sistematización de los instrumentos, es posible identificar que el diseño universal del aprendizaje como método es un campo de exploración en la primera infancia. Para el análisis en este apartado se retoman dos categorías: las concepciones frente al diseño universal como enfoque didáctico, el cual relaciona el rol del agente educativo/ docente, y el análisis frente a la implementación de los principios, relacionando el rol del niño como sujeto actor y el rol de la institución.

En cuanto al rol del agente educativo se comprenden las concepciones: se puede decir que la interpretación que hacen las agentes educativas de educación inicial y las 
docentes de los grados transición, primero y de las fundaciones en las cuales se aplicó la propuesta en relación a la comprensión y aplicación de cada uno de los principios, es proporcionar múltiples formas de representación de la información y los contenidos, así como múltiples formas de expresión del aprendizaje y múltiples formas de implicación, que aún están mediadas por concepciones teóricas e imaginarios que si bien lo centran en la atención a la diversidad desde la participación que puede tener el sujeto, también es claro que se permea por un asunto actitudinal en el que los docentes unifican los principios y centran sus miradas en el desarrollo de estrategias para representar la información o para el diseño de materiales. De esta forma, el diseño universal lo concibe como un modelo para presentar la información de acuerdo a los estilos y estrategias de los niños y niñas, es una forma de facilitar la participación y aceptación dentro de los procesos académicos, no obstante, refieren la dificultad para aplicarlo en tanto lo relacionan directamente con el diseño de materiales:

El diseño universal es proporcionar múltiples formas de aprendizaje a los niños y niñas para que ellos puedan participar y se puedan sentir aceptados, es un diseño de un currículo flexible para que todos, hasta los niños con discapacidad, puedan aprender; sin embargo, es difícil aplicarlo, no podemos estar haciendo materiales todo el tiempo, las dinámicas aquí son muy complejas, hay que avanzar en lo curricular. (Docente de grado transición)

Al indagar por el diseño universal en el marco de la educación inicial, las agentes educativas refieren desconocimiento por el tema. Sin embargo, realizan una asociación con respecto a las múltiples estrategias metodológicas y didácticas que se emplean para favorecer la participación, enuncian, además, las diferencias que existen entre los sistemas de educación inicial y formal, y determinan aspectos centrados en las prácticas pedagógicas de ambos escenarios movilizando reflexiones en cuanto a los modelos tradicionales de la escuela y las propuestas flexibles del sistema de educación inicial.

En la primera infancia no he escuchado mucho esto porque realmente nosotros utilizamos muchas estrategias flexibles para todos los niños y niñas, para que ellos participen y puedan ser escuchados, digamos que nuestros diseños son para todos, sé que en la escuela sí necesitan de este tipo de diseños porque son muy tradicionalistas y no emplean el juego u otras metodologías para mediar el aprendizaje; ahí sí necesitan de ese diseño.

(Agente educativa modalidad institucional)

Aunque es un discurso nuevo y está adaptado desde la accesibilidad, se puede decir, por ejemplo, que la institución emplea este enfoque y realmente favorece el aprendizaje de todos. Ahora bien, es cierto que no todos los docentes lo aplican en su práctica pedagógica, lo asumen en el discurso pero no son conscientes de las implicaciones que tiene, ahí es cuando no hay una verdadera realidad entre la práctica y la teoría y cuando se presenta como una barrera para el logro del aprendizaje. (Docente de grado primero, Institución Educativa) 
Bajo esta mirada es posible identificar que existen diversas configuraciones con respecto a lo que se entiende por diseño universal, si bien este se comprende como ese conjunto de principios y estrategias que favorecen las posibilidades de aprendizaje y la participación efectiva de todos los aprendices en el aula, en tanto permite al maestro orientar sus espacios pedagógicos con metodologías flexibles y alternativas que abarcan las singularidades e identidades del sujeto, y que responde a las metas y objetivos educativos, es cierto que de acuerdo con las voces de las docentes aún falta apropiarlo, discutirlo y replantearlo a partir del ejercicio educativo.

Continuando con el análisis frente al rol del niño y rol de la institución en la implementación, ${ }^{6}$ en la Tabla 1 se describen las acciones que se desarrollaron de acuerdo con cada uno de los principios, es importante resaltar que con las diversas actividades se visibilizó la participación de los niños-niñas y jóvenes, especialmente de aquellos que al inicio del proyecto nunca participaban, se aislaban o desafiaban las actividades. Se encuentra que aquellos niños y jóvenes caracterizados por sus diagnósticos lograron reconocer sus capacidades y mostrar sus talentos gracias al refuerzo positivo, a la constante motivación, a la variación de los niveles y al trabajo frente a lo emocional, la confianza y el reconocimiento de ellos mismo como sujetos importantes en el proceso de enseñanza-aprendizaje.

Tabla 1. Aplicación del diseño universal. Estrategias metodológicas y didácticas implementadas

\begin{tabular}{|c|c|c|c|c|}
\hline \multicolumn{4}{|c|}{$\begin{array}{l}\text { Aplicación del diseño universal- } \\
\text { Estrategias metodológicas y didácticas implementadas }\end{array}$} & $\begin{array}{l}\text { Logros evidenciados } \\
\text { en los niños-niñas }\end{array}$ \\
\hline \multirow{7}{*}{$\begin{array}{c}\text { Diseño } \\
\text { Universal } \\
\text { del } \\
\text { Aprendizaje }\end{array}$} & Principios & $\begin{array}{l}\text { Áreas de trabajo } \\
\text { presentes en } \\
\text { cada una de } \\
\text { las unidades } \\
\text { didácticas }\end{array}$ & $\begin{array}{c}\text { Acciones } \\
\text { desarrolladas en las } \\
\text { planeaciones }\end{array}$ & \multirow{7}{*}{$\begin{array}{l}\text { 1. Mayor reconocimiento de } \\
\text { actividades guiadas } \\
\text { 2. Mejor comprensión de los } \\
\text { conceptos } \\
\text { 3. Mayor grado de atención y } \\
\text { concentración } \\
\text { 4. Logro en las metas de } \\
\text { aprendizaje } \\
\text { 5. Claridad en el procesamiento } \\
\text { de la información } \\
\text { 6. Activación de saberes } \\
\text { 7. Creatividad- Imaginación } \\
\text { 8. Permanencia en la tarea } \\
\text { 9. Transferencia de saberes y } \\
\text { conocimientos } \\
\text { 10. Facilita la comprensión de } \\
\text { temas complejos } \\
\text { 11. Memoria } \\
\text { 12. Dominio de los contenidos }\end{array}$} \\
\hline & \multirow{6}{*}{ Representación } & \multirow{6}{*}{$\begin{array}{l}\text { Opciones para la } \\
\text { información }\end{array}$} & $\begin{array}{l}\text { Opciones lenguajes, } \\
\text { símbolos pictogramas } \\
\text { y diagramas }\end{array}$ & \\
\hline & & & Material concreto & \\
\hline & & & $\begin{array}{c}\text { Alternativas } \\
\text { visuales, auditivas, } \\
\text { táctiles, opciones de } \\
\text { percepción }\end{array}$ & \\
\hline & & & Recursos didácticos & \\
\hline & & & $\begin{array}{l}\text { Personalizar } \\
\text { presentación } \\
\text { información }\end{array}$ & \\
\hline & & & $\begin{array}{l}\text { Opciones para } \\
\text { comprensión }\end{array}$ & \\
\hline
\end{tabular}

6 Las propuestas pedagógicas implementadas en cada una de las instituciones se diseñaron con múltiples estrategias respetando las metodologías institucionales, por ello se planearon a partir de los lenguajes artísticos, los portadores de texto, la expresión dramática y las estrategias sensoriales. La planeación contó con unidades didácticas que fueron transversales a los contenidos propuestos por las docentes en sus planes de estudio y estructuradas de acuerdo a los principios del diseño universal (representación, expresión, implicación). 


\begin{tabular}{|c|c|c|c|c|}
\hline \multicolumn{4}{|c|}{$\begin{array}{l}\text { Aplicación del diseño universal- } \\
\text { Estrategias metodológicas y didácticas implementadas }\end{array}$} & $\begin{array}{l}\text { Logros evidenciados } \\
\text { en los niños-niñas }\end{array}$ \\
\hline \multirow{10}{*}{$\begin{array}{c}\text { Diseño } \\
\text { Universal } \\
\text { del } \\
\text { Aprendizaje }\end{array}$} & Principios & $\begin{array}{l}\text { Áreas de trabajo } \\
\text { presentes en } \\
\text { cada una de } \\
\text { las unidades } \\
\text { didácticas }\end{array}$ & $\begin{array}{c}\text { Acciones } \\
\text { desarrolladas en las } \\
\text { planeaciones }\end{array}$ & \multirow{7}{*}{$\begin{array}{l}\text { 1. Confianza, seguridad } \\
\text { 2. Credibilidad y escucha por el otro } \\
\text { 3. Promoción de pensamiento } \\
\text { crítico } \\
\text { 4. Desarrollo del lenguaje } \\
\text { 5. Logro en permanecer en la activi- } \\
\text { dad y terminarla } \\
\text { 6. Expresión de saberes, pensamien } \\
\text { tos, sentimientos } \\
\text { 7. Comprensión } \\
\text { 8. Capacidad de negociar } \\
\text { 9. Resiliencia, aumento de voca- } \\
\text { bulario } \\
\text { 10. Regulación de comportamientos }\end{array}$} \\
\hline & \multirow{6}{*}{ Expresión } & $\begin{array}{l}\text { Opciones de } \\
\text { lenguaje }\end{array}$ & $\begin{array}{l}\text { Opciones de } \\
\text { vocabulario y símbolos }\end{array}$ & \\
\hline & & \multirow[t]{2}{*}{$\begin{array}{l}\text { Métodos de } \\
\text { respuesta }\end{array}$} & $\begin{array}{l}\text { Interacción con los } \\
\text { materiales concretos- } \\
\text { procesamiento de } \\
\text { información }\end{array}$ & \\
\hline & & & $\begin{array}{l}\text { Herramientas } \\
\text { tecnológicas }\end{array}$ & \\
\hline & & \multirow{3}{*}{$\begin{array}{l}\text { Funciones } \\
\text { ejecutivas }\end{array}$} & $\begin{array}{l}\text { Establecimiento } \\
\text { de metas }\end{array}$ & \\
\hline & & & $\begin{array}{l}\text { Planificación y } \\
\text { desarrollo de } \\
\text { estrategias y } \\
\text { habilidades }\end{array}$ & \\
\hline & & & $\begin{array}{c}\text { Seguimiento } \\
\text { de avances- } \\
\text { autoevaluación }\end{array}$ & \\
\hline & \multirow{3}{*}{ Implicación } & $\begin{array}{l}\text { Intereses } \\
\text { personales }\end{array}$ & $\begin{array}{l}\text { Expectativas de los } \\
\text { niños-niñas }\end{array}$ & \multirow{3}{*}{$\begin{array}{l}\text { 1. Autonomía } \\
\text { 2. Seguridad y confianza } \\
\text { 3. Toma de decisiones } \\
\text { 4. Trabajo independiente } \\
\text { 5. Participación espontánea } \\
\text { 7. Esfuerzo personal y persistencia }\end{array}$} \\
\hline & & Autorregulación & $\begin{array}{l}\text { Motivación- } \\
\text { emociones }\end{array}$ & \\
\hline & & Metas y objetivos & $\begin{array}{l}\text { Variación de los } \\
\text { niveles }\end{array}$ & \\
\hline
\end{tabular}

Fuente: Elaboración propia

Después de la implementación se encuentra que en las actividades desarrolladas en el principio uno - Proporcionar múltiples medios de representación-, los niños y niñas dentro de las actividades logran tener una mejor comprensión de los temas y conceptos a partir de diversas maneras de representarlos, tales como el uso del vídeo, pictogramas, sonidos, recursos didácticos como los cuentos, organizadores visuales, definición de vocabulario y símbolos, decodificación de textos, vinculación de conceptos previos, entre otros. Adicionalmente, el empleo de los pictogramas facilita guiar las actividades de las rutinas diarias lo que posibilita un comportamiento adecuado en las clases; el dibujo y la implementación de símbolos apoyan la construcción de conceptos desde la ilustración no lingüística, lo que permite el fortalecimiento de la memoria, la transferencia y el procesamiento de la información. La organización estructural del aula desde el trabajo colaborativo y la disposición de los ambientes ayudan a que el niño se sienta más a gusto con lo que hace, permite que siga instrucciones y fortalece poco a poco su toma de decisiones; asimismo, la utilización de agendas permite a los niños el desarrollo de ideas claras sobre los temas.

En los momentos de interacción y manipulación con los elementos didácticos, los niños tuvieron mejor reconocimiento por las actividades guiadas que se les presentaban. En la realización de las diferentes secuencias en las que tenían que manipular, construir y sentir demostraron empatía, gusto y felicidad, lo que ocasionaba que cada vez 
que se realizaba alguna actividad su motivación fuera más alta y tuvieran una mejor comprensión hacia los contenidos. Por otra parte, también se logró visualizar la atención y concentración que lograban durante las actividades. Cabe resaltar que a los niños que se les dificultaba tener una concentración en los temas dirigidos por la docente, demostraron un gran cambio y mejoría en su atención, un ejemplo de ello es que seguían las instrucciones, terminaban las actividades y participaban sin desertar de la actividad.

Es necesario destacar la importancia que pintar, dibujar, tocar un instrumento musical, modelar, cantar, interactuar, escuchar música y audios y ver agendas visuales e imágenes tienen en los niños del grado primero o con extra edad, en tanto hay una mejor respuesta al proceso de aprendizaje, y esto se evidencia en la manera en que interpretan y le dan sentido a cada actividad, los niños con alertas en el desarrollo y con discapacidad logran participar e implicarse en las actividades y lo más significativo: responder desde sus habilidades y capacidades en cada uno de los ejercicios. Se debe mencionar, no obstante, que sigue haciendo falta emplear mapas semánticos, hacer énfasis en aspectos como proporcionar alternativas para definir vocabularios y símbolos, aclarar sintaxis, decodificar textos y la notación matemática. Los resultados en el principio Múltiples medios para la Acción y la Expresión, se evidencian en las manifestaciones de los niños y las niñas, la expresión de seguridad, participación y liderazgo para expresar sentimientos, emociones, saberes, preguntas y cuestionamientos sin temor a ser rechazados fueron las principales características de los encuentros. Facilitarles diferentes maneras de respuesta, entre ellas oral, escrita, diálogos, dibujos y expresiones con el cuerpo, les genera confianza, seguridad y autonomía. Asimismo, el establecimiento de metas de acuerdo con los niveles cognitivos y las realizaciones de actividades para movilizar las funciones ejecutivas, se constituyen en estrategias fundamentales para el seguimiento en los aprendizajes, la promoción de pensamiento crítico, el desarrollo del lenguaje, la permanencia en la actividad y la capacidad de negociar.

En el principio tres, Múltiples medios para la motivación e implicación en el aprendizaje, las actividades estuvieron orientadas a que los niños y niñas desarrollaran la autonomía y la autorregulación; algunos niños y niñas presentan dificultades para controlar emociones y manifiestan expresiones de ira y autoagresión. No obstante, con las estrategias se lograron vincular a los procesos, participar de forma espontánea, persistir en el logro de las actividades y autorregular comportamientos. Se resalta que en el trabajo a la mayoría de los niños les motiva lo novedoso, mientras que otros prefieren los procesos rutinarios. Hay niños que se concentran mejor cuando trabajan solos; en otros casos, prefieren trabajar en grupo. Por ello es importante dar opciones o variar los niveles de exigencia de modo que todos puedan implicarse según sus preferencias. 


\section{Discusión de los resultados}

La primera infancia es un período de la vida reconocido universalmente por sus transformaciones en el ciclo de vida, porque sienta las bases para el desarrollo de la inteligencia y la personalidad, en tanto se obtiene el máximo desarrollo del cerebro, del lenguaje y del pensamiento; es una etapa en la que el niño obtiene independencia, identidad, autonomía y confianza para construir su historia de vida. Son varias las construcciones epistemológicas y pedagógicas que ponen las infancias, la institucionalidad y la enseñanza como punto de discusión en los procesos de enseñanza-aprendizaje. Algunos autores en sus trabajos consideran que la atención a la diversidad es un punto de debate en correspondencia con la inclusión, la exclusión, la interseccionalidad, los currículos, la enseñanza y el aprendizaje (Jean Louis, 1976; Donzelot, 1979; R. Blanco, 1999, 2006; M. Blanco, 2008; Dussel, 2004; Fernández, 2003; Correa y Restrepo, 2018). Para Adriana Puiggros (1990, p. 234), la educación constituye un producto y una práctica social compleja, y una de estas complejidades se da en la búsqueda por "facilitar y brindar oportunidades efectivas de aprendizaje a cada niño, niña y adolescente, y concebir la diversidad como la oportunidad para propiciar espacios educativos en los que se respete, valore y responda a la diversidad" (Unesco, 2008).

Carlos Parra (2010, p. 77) anota que pese a los dilocuentes discursos entre integración e inclusión los 'otros' 'discapacitados' 'diferentes' continúan invisibilizados en la escuela. Un dato estadístico del Banco Mundial 2004 revela que:

Sólo entre el 20\% y el 30\% de los niños y niñas con discapacidad asisten a la escuela. Según los datos disponibles, puede concluirse que los niños y niñas con discapacidad suelen ser excluidos de los sistemas educativos. En Colombia, sólo el 0,32\% de los alumnos que asisten a la escuela tienen una discapacidad promedio muy por debajo del porcentaje de niños y niñas con discapacidad en el país, de acuerdo a los datos del Monitoreo Internacional de los Derechos de las Personas con Discapacidad (2004). El acceso a la educación varía según el tipo y el grado de discapacidad, y la gran mayoría de las matrículas se concentran en la educación primaria. (OMS y Banco Mundial, 2011)

De acuerdo al último informe de la OCDE (2016), en Colombia la situación actual con respecto a la oferta educativa para los niños y las niñas menores de seis años muestra marcadas diferencias en cuanto al acceso a oportunidades, aspectos como la cobertura, los procesos de aprendizaje y enseñanza y el acceso a servicios de atención especialmente para las poblaciones en las zonas rurales y niños y niñas con necesidades educativas y alertas en el desarrollo, muestran índices de participación inferiores y menos logros académicos, por ello se conciben dos retos fundamentales: cerrar las brechas existentes en términos de participación y mejorar la calidad de la educación para todos. 
En este sentido, el proceso de acceso y permanencia en el sistema de educación infantil y formal evidencia una ruptura frente a la trayectoria. El reconocimiento y la valoración de las identidades y singularidades de los sujetos y la diversidad en el aula representan un reto para la enseñanza. De acuerdo con el Ministerio de Educación Nacional (MEN) el elemento primordial en el proceso de enseñanza y aprendizaje es el diseño y la implementación de acciones y estrategias institucionales, pedagógicas y didácticas, en las que todos y cada uno de los estudiantes accedan al aprendizaje con facilidad (MEN, 2016). José Contreras establece que "no existen dos alumnos que sean iguales, precisamente porque en eso reside la riqueza de lo humano, en la diversidad y singularidad de cada sujeto" (2002).

Por ello, para mediar la participación en el país se han propuesto múltiples alternativas entre ellas el diseño universal del aprendizaje, el cual busca que el "docente transforme el aula y la práctica pedagógica, facilite la evaluación y seguimiento a los aprendizajes" (MEN, 2017). Elizabeth Hartmann enuncia que el DUA es una nueva manera de pensar la educación, “es una filosofía, que tiene el potencial para reformar el currículo y hacer que las experiencias de aprendizaje sean más accesibles y significativas” para la población estudiantil en general (2011, p. 1). Pastor, Sánchez y Zubillaga (2011) relacionan que el (DUA) ayuda a tener en cuenta la variabilidad de los estudiantes al sugerir flexibilidad en los objetivos, métodos, materiales y evaluación.

Así, se puede decir que el diseño se convierte en un eje fundamental para la enseñanza, pues se comprende como un enfoque multidimensional que posibilita la implementación de variadas didácticas y estrategias metodológicas que fomentan la participación, el reconocimiento y la implicación de los sujetos, además reconoce la singularidad e identidad en tanto visualiza la flexibilidad en los contenidos, objetivos, métodos y evaluaciones, poniendo al sujeto en el centro de la enseñanza. Marín, Roldán y Restrepo (2018) afirman que la implementación del diseño universal contrarresta las metodologías tradicionales conductistas que hacen que el estudiante cumpla un papel pasivo, y permite situar al sujeto dentro del proceso de aprendizaje no como niño, niña, joven (discapacitado, afro, pobre) necesitado y carente, sino como sujeto de aprendizaje con capacidades.

No obstante, es preciso decir que su implementación se ve mediada por algunos retos pues su conceptualización y apropiación requieren mayor discusión en el campo educativo. De acuerdo a la investigación realizada por Andrade (2017), los maestros reconocen la importancia del DUA y del deber que tienen como educadores de implementar de manera eficaz este tipo de propuestas dentro de las actividades, sin embargo, los docentes manifiestan desconocimiento de las guías que son enviadas por el Ministerio de Educación. Sánchez y Díez (2013) establecen que las pautas del DUA son la base para transformar los currículos. Empero, no deberían utilizarse como un "recetario", sino más bien como un conjunto de estrategias que se pueden emplear para superar las barreras inherentes a gran parte de los currículos existentes. 
Poniendo en discusión el rol del agente educativo versus el rol del niño como actor fundamental en el proceso de aprendizaje y la institución como mediadora en la enseñanza, y comprendiendo que en ocasiones se tiende a discriminar a los estudiantes con un capital distinto al dominante o se brindan atenciones que garantizan el acceso, pero no el acompañamiento en la trayectoria escolar y la transición del sujeto de la educación inicial a la educación formal en Colombia, y en pro de trascender lo metodológico y pedagógico, se proponen las siguientes reflexiones frente a la implementación del diseño universal y la implicación que tiene no solo para los docentes, agentes educativos, sino para las instituciones como escenarios educativos incluyentes (Tabla 2).

Tabla 2. Diseño universal del aprendizaje. Implementación/ Implicación?

\begin{tabular}{|c|c|}
\hline \multicolumn{2}{|c|}{ Rol del agente educativo/ docente- Rol de la institución } \\
\hline Implicaciones: Diversidad & $\begin{array}{c}\text { Implementar: acompañamiento Transiciones } \\
\text { Educativas }\end{array}$ \\
\hline $\begin{array}{l}\text { Comprender la diversidad en el contexto } \\
\text { educativo infantil implica educar para el } \\
\text { pluralismo y conlleva enseñar desde la } \\
\text { equiparación de oportunidades, reflejadas } \\
\text { no solo en el acceso, sino en los procesos } \\
\text { de aprendizaje. Es claro que todos los niños } \\
\text { pueden aprender, pero no todos aprenden } \\
\text { de la misma forma, asimismo, no existen } \\
\text { dos estudiantes que sean iguales, atender la } \\
\text { diversidad resulta para el docente un reto } \\
\text { en tanto no solo debe reconocer al sujeto } \\
\text { aprendiz como sujeto de saber, con capacidad } \\
\text { de agenciamiento, sino como un sujeto que se } \\
\text { intersecciona por aspectos sociales, culturales, } \\
\text { cognitivos y emocionales, que interfieren en la } \\
\text { identidady trayectoria escolar del sujeto, lo que } \\
\text { sin duda es fundamental para que el docente o } \\
\text { agente educativo pueda comprender la historia } \\
\text { de vida, las biografías y direccionar acciones } \\
\text { y estrategias institucionales, pedagógicas } \\
\text { y didácticas que favorezcan el acceso al } \\
\text { aprendizaje de forma pertinente y fácil. }\end{array}$ & $\begin{array}{l}\text { En la educación inicial las prácticas y los saberes en } \\
\text { los diferentes entornos se caracterizan por poner al } \\
\text { estudiante como eje central, por ello se proponen } \\
\text { ambientes potenciadores de aprendizaje que } \\
\text { buscan favorecer el desarrollo del pensamiento, y } \\
\text { las habilidades cognitivas, sociales, emocionales, } \\
\text { y comunicativas de todos los niños y niñas. Las } \\
\text { propuestas pedagógicas del sistema de atención } \\
\text { integral y el grado preescolar se transversalizan por } \\
\text { los pilares como el juego, el arte, la literatura y la } \\
\text { exploración del medio, y son motivantes en tanto } \\
\text { suscitan el asombro, la curiosidad, la pregunta, la } \\
\text { activación de saberes previos, la creatividad y la } \\
\text { autonomía. No obstante, hay una diferencia cuando } \\
\text { el niño-niña transita al sistema de educación } \\
\text { formal básica primaria, porque las metodologías } \\
\text { cambian, los ritmos son diferentes y son los } \\
\text { niños los que se ven enfrentados a barreras en los } \\
\text { contenidos, currículos, objetivos y evaluaciones a } \\
\text { los cuales se deben adaptar. Las transiciones para } \\
\text { los niños con discapacidad, alertas en el desarrollo, } \\
\text { intersección etnia-cultura, condición económica y } \\
\text { social es un proceso complejo y crítico que implica } \\
\text { la adaptación y asimilación a un nuevo modelo, con } \\
\text { características diferentes y que se va complejizando } \\
\text { en la medida en que van avanzando de un nivel a } \\
\text { otro, hay que decir que para la educación inicial y } \\
\text { formal este es un desafío que requiere no solo la } \\
\text { articulación interinstitucional, el diálogo entre los } \\
\text { dos escenarios, sino que implica mirar la transición } \\
\text { desde la trayectoria escolar para poder hacer un } \\
\text { efectivo acompañamiento en el curso de vida. }\end{array}$ \\
\hline
\end{tabular}

7. Esta construcción hace parte de la ponencia "Retos y desafíos en la educación inicial" presentada en el marco del IX Congreso internacional de discapacidad realizado en la ciudad de Medellín (2018). 
Rol Agente educativo/docente- Rol de la Institución

\begin{tabular}{|l|}
\hline \multicolumn{2}{|c|}{ Implicación: Prácticas Pedagógicas } \\
\hline $\begin{array}{l}\text { Comprender que las prácticas pedagógicas } \\
\text { requieren mayor reflexión y la articulación }\end{array}$
\end{tabular}
entre las metodologías implementadas en el sistema de atención integral versus el sistema de educación formal, debe reconfigurar nuevas construcciones conjuntas que permitan alternar metodologías flexibles y alternativas que contrarresten las estrategias de enseñanza tradicionales, conductistas, homogéneas y las pedagogías que no dan respuestas a las singularidades, y que además se colonicen las construcciones que hay del niño con discapacidad o alertas en el desarrollo como un sujeto carente o con necesidad. El proceso educativo se debe centrar en apropiar un enfoque de capacidades en tanto se propone como elemento de la justicia social, pues se respeta la diversidad, la dignidad y la autonomía de cada uno de los individuos, ayudando a centrar la mirada en las fortalezas y las potencialidades de los niños y niñas. Las capacidades también deben ser entendidas como los diversos funcionamientos de una persona, es decir, no se comprenden como una sola habilidad pasiva sino como una destreza que cambia, se transforma, y que incluye todas las posibilidades de actuación en los diferentes contextos.

Hay que decir que si se asume esta perspectiva, los funcionamientos logrados por un individuo o un grupo cultural pueden permitir el agenciamiento $o$ empoderamiento para determinar la calidad de vida y el bienestar de los sujetos, y es ahí donde se deben consolidar los currículos y las propuestas pedagógicas, pues lo que se requiere para equiparar oportunidades y garantizar la participación es el empoderamiento.

Implementar Diseño Universal del Aprendizaje

Responder a la Justicia curricular y los ajustes razonables desde los marcos curriculares es una de las exigencias en el marco de las normatividades para la educación infantil y formal; lograr la participación efectiva de los niños y niñas, con o sin discapacidad, implica diferentes ajustes durante el proceso educativo en cuanto a la accesibilidad y el currículo para eliminar obstáculos y barreras.

Aplicar e implementar el diseño universal del aprendizaje (DUA) para que todos los estudiantes independientemente de sus individualidades puedan acceder sin discriminación, permite el ejercicio transversal de los procesos educativos; en tanto aplicado a la educación, alternando en simultánea los tres principios - representación, expresión, implicación-, forja metas y objetivos que realmente fomentan aprendices expertos, autónomos y seguros del saber capaces de responder a las exigencias y necesidades académicas.

En el marco de la enseñanza, su fundamento en el funcionamiento cognitivo frente a la forma de acceder y percibir la información, la forma de expresar los saberes con relación a las habilidades estratégicas y organizativas de cada individuo, y la red emocional y afectiva frente al proceso y disposición del sujeto para aprender en correspondencia con la motivación, el esfuerzo personal, la persistencia y la implicación en el proceso de aprendizaje, contribuye significativamente en el empoderamiento de los niños y niñas, y el desarrollo de las habilidades para la vida (habilidades sociales, cognitivas y para el control de las emociones). 
Rol Agente educativo/docente- Rol de la Institución

\begin{tabular}{|c|c|}
\hline \multicolumn{2}{|c|}{ Rol Agente educativo/docente- Rol de la Institución } \\
\hline Implicaciones Aprendizajes & Implementar DUA: Calidad de los Saberes \\
\hline $\begin{array}{l}\text { Comprender que los aprendizajes no son rutinarios } \\
\text { ni repetitivos, que se deben construir para la vida y } \\
\text { que por ello resulta indispensable el desarrollo de } \\
\text { habilidades adaptativas, sociales, cognitivas y para } \\
\text { el control emocional. Se hace necesario mediar } \\
\text { los currículos con propuestas educativas que } \\
\text { reconozcan a los sujetos como seres integrales, } \\
\text { singulares con historias propias, con capacidades, } \\
\text { habilidades y actitudes, capaces de transformar } \\
\text { su propio aprendizaje en coherencia con sus } \\
\text { singularidades e identidades. } \\
\text { Las metas de aprendizaje se deben centrar en } \\
\text { correspondencia con los niveles de desarrollo } \\
\text { de los niños y niñas y esto no implica para los } \\
\text { docentes mayor trabajo, refiere una configuración } \\
\text { del currículo con ajustes que visualicen objetivos } \\
\text { pertinentes y diferentes metas de respuestas, } \\
\text { variaciones en los niveles de desafío y en las } \\
\text { opciones y dinámicas de trabajo, además de la } \\
\text { minimización de las inseguridades, pues estas son } \\
\text { las que en ocasiones no permiten la participación } \\
\text { de los niños y niñas. }\end{array}$ & $\begin{array}{l}\text { Los saberes, teniendo presente el diseño universal, } \\
\text { se deben considerar en el marco del diálogo } \\
\text { igualitario, donde los intercambios de experiencias } \\
\text { en las interacciones propicien un aprendizaje } \\
\text { continuo permanente mediado por la validación y } \\
\text { el reconocimiento del otro. Hay que fortalecer la } \\
\text { autoimagen y la autoestima, generar en los niños } \\
\text { y niñas confianza, seguridad y empatía para que } \\
\text { puedan lograr las metas de aprendizaje propuestas. } \\
\text { Trabajar en el marco de la solidaridad, así todos } \\
\text { pueden aportar sin competitividad, buscando } \\
\text { la confianza, el apoyo mutuo y la no imposición, } \\
\text { se trata de aprender de todos y para todos en un } \\
\text { intercambio de conocimientos y experiencias, por } \\
\text { ello se debe propender por saberes prácticos, pero } \\
\text { además saberes que involucren el trabajo con las } \\
\text { emociones y el desarrollo de la comunicación no } \\
\text { verbal, este último es trascendental para reconocer } \\
\text { sentimientos, capacidades, silencios, expresiones, } \\
\text { llantos y angustias, eje fundamental para } \\
\text { interpretar las múltiples maneras en que expresan } \\
\text { lo que saben y las diversas formas en que se van a } \\
\text { motivar e implicar en su propio aprendizaje. }\end{array}$ \\
\hline
\end{tabular}

Rol del Niño

\begin{tabular}{|c|c|}
\hline Participación & Autonomía \\
\hline $\begin{array}{l}\text { Se debe mencionar, de igual modo, que el trabajo } \\
\text { con los niños y niñas que presentan intersección } \\
\text { por discapacidad, alerta en el desarrollo, situación } \\
\text { social por pobreza, desplazamiento, conflicto } \\
\text { armado, entre otros, debe ante todo promover el } \\
\text { desarrollo del autorreconocimiento como sujetos, } \\
\text { se debe propiciar un entorno de aula en el cual } \\
\text { puedan desarrollar su autonomía y autorregulación, } \\
\text { posibilitar que el estudiante se pueda vincular de } \\
\text { manera más asertiva en los procesos cotidianos } \\
\text { del aula, y que establezca rutinas estables que le } \\
\text { permitan alcanzar metas efectivas. }\end{array}$ & $\begin{array}{l}\text { Hacer uso del diseño universal contribuye a } \\
\text { construir la personalidad del niño y la niña, } \\
\text { potenciando las habilidades y capacidades } \\
\text { individuales, comotambién fortalece la creatividad } \\
\text { en tanto se brinden diferentes oportunidades; } \\
\text { permitirles tomar decisiones, autoevaluar y hacer } \\
\text { parte de su proceso individual, reconocerse como } \\
\text { propios, como seres autónomos y por ende incidir } \\
\text { en su autorregulación. A su vez, cuando el niño } \\
\text { tiene credibilidad avanza en el proceso académico. }\end{array}$ \\
\hline
\end{tabular}

Fuente: Elaboración Propia

\section{Conclusiones}

A modo de cierre, la cobertura para los programas de educación inicial en respuesta a la atención a la diversidad implica una atención diferenciada en la que se potencie el desarrollo integral y el reconocimiento de los intereses y particularidades personales, culturales y sociales de las niñas, niños y sus familias, así como las características y las condiciones de los contextos en donde viven, lo que exige para el profesional en este campo de saber tener una formación en atención a la diversidad y el desarrollo 
de altas competencias pedagógicas para el trabajo con poblaciones diversas. Asimismo, implica tener apertura y una mirada intercultural que posibilite poner al otro en equiparación de oportunidades y desarrollar situaciones de aprendizaje que potencialicen y fortalezcan el desarrollo de habilidades a lo largo de la vida, se debe trabajar en los procesos de formación desde un enfoque etno-relativo, donde se pueda transformar paradigmas y actitudes en relación con la interculturalidad, la diversidad y la inclusión, y propuestas pedagógicas que apropien los principios del diseño universal del aprendizaje, las metodologías flexibles y las estrategias organizativas.

El diseño universal del aprendizaje como método es un campo de exploración en la primera infancia. La comprensión y aplicación de cada uno de los principios aún están mediadas por concepciones teóricas e imaginarios que si bien centran el DUA en la atención a la diversidad desde la participación que puede tener el sujeto, también es claro que se permea por un asunto actitudinal. Es importante analizar los discursos de atención a la diversidad que hay alrededor de la diversidad misma, las identidades, las infancias y la inclusión en las prácticas educativas de la educación infantil, para comprender los estigmas y subjetividades y desarrollar propuestas que realmente reconozcan las identidades y pluralidades de los sujetos. Es importante, además, entender las biografías de los niños y niñas en tanto hay situaciones que interfieren en los procesos de aprendizaje y se relacionan no solo con las condiciones sociales y culturales, sino con las oportunidades y los reconocimientos que los sujetos tienen en su proceso de aprendizaje.

Se debe reflexionar sobre cómo se asumen los propósitos y principios de la educación inclusiva. No en todas las propuestas educativas y los currículos se tienen en cuenta los ajustes razonables y la singularidad de los sujetos, en ocasiones se tiende a discriminar a los estudiantes con un capital distinto a la dominante, o se brindan atenciones que garantizan el acceso, pero no el acompañamiento en la trayectoria escolar y la transición. Se debe reflexionar también sobre cómo se conciben las infancias, la educación, la institucionalidad, los discursos y la forma como definimos al otro, cómo nos relacionamos con el otro y cómo lo posicionamos en los procesos educativos.

\section{Referencias}

Alba, C., Sánchez, J. y Zubillaga, A. (2011). Diseño Universal para el Aprendizaje (DUA). Pautas para su introducción en el currículo. España: DUALETIC. Recuperado de http://www. educadua.es/doc/dua/dua_pautas_intro_cv.pdf

Correa A., J. I. y Restrepo R., N. (2018). Atención a la diversidad: retos y desafíos en la Educación Superior. En J. C. Tovar (Ed.), Trends and challenges in Higher Education in Latin America (pp. 179-190). Eindhoven, NL: Adaya Press.

Blanco, M. (2008). Construyendo las bases de inclusión y la calidad de la educación en la primera infancia [Internet]. Revista de Educación, (347), 33-54. Recuperado de http://www.revistaeducacion.mec.es/re347/ re347_02.pdf 
Blanco, R. (1999). Hacia una escuela para todos y con todos. Boletín del Proyecto Principal de Educación para América Latina y el Caribe, (48), 57-72. Santiago de Chile: Unesco.

Blanco, R. (2006). La equidad y la inclusión social: uno de los desafíos de la educación y la escuela hoy. REICE: Revista Electrónica Iberoamericana sobre Calidad, Eficacia y Cambio en Educación, 4(3), 1-15. Recuperado de http:// www. rinace.net/arts/vol4num3/art1.pdf

Berquist, L. \& Moore, J. (marzo, 2014). Using UDL exchange to teach the universal design for learning framework to pre-service teachers. In M. Searson \& M. Ochoa (Eds.), Proceedings of Society for Information Technology \& Teacher Education International Conference (SITE 2014) (pp. 6-7). Chesapeake, VA.

Congreso de la República de Colombia (2 de agosto de 2016). Ley 1804 de 2016. "Por la cual se establece la Política de Estado para el Desarrollo Integral de la Primera Infancia de Cero a Siempre y se dictan otras disposiciones. Recuperado de https://www.mineducacion.gov.co/1759/w3-article-381611.html

Congreso de la República de Colombia (27 de febrero de 2013). Ley 1618 de 2013. "Por medio de la cual se establecen las disposiciones para garantizar el pleno ejercicio de los derechos de las personas con discapacidad". Recuperado de http:/wsp.presidencia.gov.co/Normativa/Leyes/Documents/2013/LEY\%20 1618\%20DEL\%2O27\%20DE\%2OFEBRERO\%2ODE\%2O2O13.pdf

Contreras, J. (1994). La investigación en la acción: ¿Qué es? Cuadernos de pedagogía, (224), 8-12.

Contreras, J. (2002). Educar la mirada... y el oído. Percibir la singularidad y también las posibilidades. Cuadernos de Pedagogía, (311), 61-65. Barcelona. España

Donzelot, J. (1979). La policía de las familias. Valencia: Ed. Pre-textos.

Dussel, I. (2004). Inclusión y exclusión en la escuela moderna argentina: una perspectiva postestructuralista. Cadernos de Pesquisa, 34(122), 305-335.

Fernández, A. (2003). Educación inclusiva: Enseñar y aprender entre la diversidad. Revista Digital UMBRAL, (13), 1-10.

Hartmann, E. (2011, septiembre). Diseño Universal para Aprendizaje (ULD). Perspectiva de Práctica. Destacando la información sobre sordoceguera, (8). Consorcio Nacional sobre Sordoceguera. Recuperado de: http://www.perkinsla. org/recursosbiblio/_1UDL-spanish.pdf

Johnson-Harris, K. \& Mundschenk, N. (2014). Working effectively with students with $\mathrm{BD}$ in a general education classroom: The case for universal design for learning. The clearing house. Journal of Educational Strategies. Issues and Ideas, 87(4), 168-174. doi: 10.1080/00098655.2014.897927

Katz, J. (2014). Implementing the three block model of universal design for learning: Effects on teachers' self-efficacy, stress, and job satisfaction in inclusive classrooms K-12. International Journal of Inclusive Education, 8(12), 5-33. doi: 10.1080/13603116.2014.881569 
Marín, L. F. C., Roldán, K. O. y Restrepo, N. R. (2018). Prácticas pedagógicas de las maestras de educación preescolar con población diversa. Palobra: Palabra que obra, (18), 156-173.

Ministerio de Educación Nacional (MEN) (2013). Fundamentos políticos, técnicos y de gestión desde la estrategia de la atención a la primera infancia. Recuperado de: http://www.deceroasiempre.gov.co/QuienesSomos/ Documents/Fundamientos-politicos-tecnicos-gestion-de-cero-a-siempre.pdf

Ministerio de Educación Nacional de Colombia (MEN) (2015). “OECD-Colombia education and skills accession policy review: Country background report". Bogotá: MEN.

Ministerio de Educación Nacional de Colombia (MEN) (2016). Documento de orientaciones técnicas, administrativas y pedagógicas para la atención educativa a estudiantes con discapacidad en el marco de la educación inclusiva. Bogotá: Ministerio de Educación Nacional - Fundación FES.

Ministerio de Educación Nacional de Colombia (MEN) (29 de agosto 2017). Decreto 1421 de 2017. "Por el cual se reglamenta en el marco de la educación inclusiva la atención educativa a la población con discapacidad".

Oquendo, R. Á. y Benavides, D. C. (2017). Estrategias didácticas para la incorporación del diseño universal para el aprendizaje en la escuela rural. Panorama, 11(21), 69-81.

Organización de Estados Iberoamericanos (OEI) (2010) Metas educativas 2021. La educación que queremos para la generación de los bicentenarios, Documento final, Madrid

Organización Mundial de la Salud (OMS) y Banco Mundial (2011). Informe mundial sobre la discapacidad. Recuperado de: https://www.who.int/disabilities/world_ report/2011/summary_es.pdf

Parra D., C. (2010). Educación inclusiva: un modelo de educación para todos. Revista ISEES: Inclusión Social y Equidad en la Educación Superior, (8), 73-84.

Pastor, C., Sánchez, J. y Zubillaga, A. (2011). Diseño Universal para el Aprendizaje (DUA). Pautas para su introducción en el currículo. Recuperado de https:// www.educadua.es/doc/dua/dua_pautas_intro_cv.pdf

Presidencia de la República de Colombia, Consejería Presidencial para la Primera Infancia (2013). Estrategia de Atención Integral a la Primera Infancia. Fundamentos Políticos, Técnicos y de Gestión. Recuperado de http://www. deceroasiempre.gov.co/QuienesSomos/Documents/Fundamientos-politicostecnicos-gestion-de-cero-a-siempre.pdf

Puiggros, A. (1990). Sujetos, Disciplina y Curriculum en los orígenes del sistema educativo argentino. Buenos Aires: Editorial Galerna.

Sánchez, S. y Díez, E. (2013). La educación inclusiva desde el currículum: el Diseño Universal para el Aprendizaje. En H. Rodríguez y L. Torrego (coords.), Educación inclusiva, equidad y derecho a la diferencia (pp. 107-119). España: Wolters Kluwer. 
Sánchez, S., Díez, E. y Martín, R. (2015). El diseño universal como medio para atender a la diversidad en la educación. Una revisión de casos de éxito en la universidad. Contextos Educativos. Revista de Educación, o(19), 121-131. doi: http://dx.doi.org/10.18172/con.2752

Unesco (2015). Educación 2030. Declaración d’Incheon y Marco de acción. Hacia una educación inclusiva y equitativa de calidad y un aprendizaje a lo largo de la vida para todos. Recuperado de http://www.redem.org/wp-content/ uploads/2016/10/Educaci\%C3\%B3n-2030.pdf

Unesco (1990). Declaración Mundial sobre Educación para Todos: La satisfacción de las necesidades básicas de aprendizaje. Declaración de Jomtien. Nueva York: Unesco.

Unesco (2008). La educación inclusiva: el camino hacia el futuro. Conferencia Internacional de Educación. Cuadragésima octava reunión. Ginebra.

Unicef (1990). Cumbre Mundial en Favor de la Infancia, México: Primera reunión de evaluación y seguimiento. México: Editorial Sistema Nacional para el Desarrollo Integral de la Familia (DIF). Recuperado de http://www.redalyc. org/pdf/106/10632617.pdf 